\title{
Cystic lymphangioma in the peripheral jejunal mesentery in an adult and excision with laparoscopic-assisted surgery: a case report
}

Hideki Nagano ${ }^{1 *} \mathbb{D}$, Toshihisa Kimura ${ }^{1}$, Atsushi lida ${ }^{1}$, Tamotsu Togawa', Takanori Goi ${ }^{2}$ and Yasunori Sato ${ }^{3}$

\begin{abstract}
Background: Lymphangiomas are uncommon congenital malformations that present mainly in the head, neck, and axillar regions in pediatric patients. Mesenteric cystic lymphangiomas (MCLs), which occasionally present with substantial growth and the invasion of adjacent vital structures, are rarely reported in adults. We report a case of $M C L$ in an adult who was treated with laparoscopic-assisted excision.

Case presentation: A 40-year-old Japanese man visited his family physician for prolonged periumbilical pain. Plain computed tomography (CT) showed a low-density mass in his left abdomen, and he was referred to our hospital 2 weeks later. His abdomen was flat and soft, and no mass was felt upon palpation. Routine laboratory data showed no abnormalities in the blood cell counts. The levels of tumor markers, such as carcinoembryonic antigen (CEA), carbohydrate antigen 19-9 (CA19-9), and cancer antigen 125 (CA125), were within normal ranges. Contrast-enhanced CT was performed, and a low-density mass was observed with an irregular outline and poor contrast, as well as involvement of the peripheral mesenteric artery and partial compression of the adjacent jejunum without dilatation of the oral side of the bowel. The patient was diagnosed with lymphatic cysts and observed for 1 month without symptom exacerbation. Follow-up CT showed no increase in the size of the mass but showed apparent invasion of the jejunal wall without bowel obstruction. Magnetic resonance imaging (MRI) showed intermediate intensity on T1weighted imaging (T1WI) and high intensity on T2-weighted imaging (T2WI). The coronal view on T2WI clearly showed an accumulation of cystic lesions. We performed tumor excision with partial resection of the jejunum in a laparoscopicassisted manner. Pathological examination showed multicystic lesions with an attenuated endothelial lining, surrounding rich adipose tissue and scattered smooth muscle fibers; the patient was diagnosed with MCL. Immunohistochemical assays supported this diagnosis.
\end{abstract}

Conclusions: This is rare case of MCL presenting in an adult who underwent successful laparoscopic-assisted resection. Mesenteric lymphangioma (ML) should be considered in the differential diagnosis of patients with intraabdominal cysts. Radical excision is optimal, even when the patient is asymptomatic. Laparoscopic-assisted tumor resection is a suitable surgical method for treating MLs located in the peripheral mesentery.

Keywords: Mesenteric lymphangioma, Mesenteric cystic lymphangioma, Jejunum, Laparoscopic-assisted surgery, CD31, Factor VIII-related antigen, D2-40, CD34

\footnotetext{
* Correspondence: naganohideki@nhofukui.org

${ }^{1}$ Department of Surgery, National Hospital Organization Tsuruga Medical

Center, 33-1, Sakuragaoka, Tsuruga, Fukui 914-0195, Japan

Full list of author information is available at the end of the article
}

(C) The Author(s). 2019 Open Access This article is distributed under the terms of the Creative Commons Attribution 4.0 International License (http://creativecommons.org/licenses/by/4.0/), which permits unrestricted use, distribution, and reproduction in any medium, provided you give appropriate credit to the original author(s) and the source, provide a link to the Creative Commons license, and indicate if changes were made. The Creative Commons Public Domain Dedication waiver (http://creativecommons.org/publicdomain/zero/1.0/) applies to the data made available in this article, unless otherwise stated. 


\section{Background}

Mesenteric lymphangiomas (MLs) are known to be uncommon congenital malformations of the lymphatic system [1]; $65 \%$ of MLs are present at birth, and $90 \%$ of all patients are diagnosed before the age of 2 [2]. Over $95 \%$ of lymphangiomas are found in the head, neck, and axillary regions. Isolated small bowel lesions are observed in less than $1 \%$ of cases [3] but account for $70 \%$ of all intraperitoneal lymphatic tumors [4]. MLs can infiltrate the surrounding organs and cause potentially life-threatening complications, such as traumatic rupture, anemia secondary to intraabdominal or intracavitary bleeding, ischemic tissue necrosis, intestinal gangrene secondary to volvulus, and intermittent intestinal obstruction $[5,6]$. MLs occasionally grow and involve main vessels or vital structures and thus become unresectable [7]. In addition, MLs can occur not only in the root of the intestinal mesentery but also in the peripheral part adjacent to the intestinal wall [6]. MLs are traditionally classified into three types: capillary, cavernous, and cystic [8]. Herein, we report a case of mesenteric cystic lymphangioma (MCL) in a 40-yearold man who was successfully treated via laparoscopicassisted excision.

\section{Case presentation}

A 40-year-old Japanese man experienced periumbilical pain since November 2013, and although the symptoms were mild, they were prolonged. He visited his family physician in January 2014, who noted mild tenderness in the lower left quadrant. The patient underwent an abdominal CT scan. CT revealed a low-density mass that measured $43 \times 40 \mathrm{~mm}$ in size in the left abdomen. He was referred to the National Hospital Organization Tsuruga Medical Center in February. His past medical and family histories were unremarkable. On examination, the patient had a height of $179.0 \mathrm{~cm}$, a body weight of $98.7 \mathrm{~kg}$, and a body mass index (BMI) of 30.8 and did not present with anemia, icterus, edema, or malnutrition. His abdomen was flat and soft, with mild tenderness in the lower left quadrant on palpation; however, the mass could not be felt.

The laboratory results showed no abnormalities in his blood cell counts; however, an elevation in the serum alanine transaminase (ALT; $60 \mathrm{IU} / \mathrm{L}), \gamma$-glutamyl transferase ( $\gamma$-GTP; $108 \mathrm{IU} / \mathrm{L}$ ), total bilirubin (T-Bil; $1.31 \mathrm{mg} / \mathrm{dL}$ ), and total cholesterol (T-Chol; $229 \mathrm{mg} / \mathrm{dL}$ ) levels was observed. The levels of epithelial tumor markers, such as carcinoembryonic antigen (CEA; $2.1 \mathrm{ng} / \mathrm{mL}$ ), carbohydrate antigen 19-9 (CA19-9; 8.7 U/mL), and cancer antigen 125 (CA125; $11 \mathrm{U} / \mathrm{mL}$ ), were within normal limits.

The patient underwent a contrast-enhanced CT examination 2 weeks after the CT examination conducted by his family physician because in the previous examination, the patient had not been administered a contrast agent. A low-density mass with an irregular outline and measuring $45 \times 42 \mathrm{~mm}$ in size was detected in the left abdomen at the slightly cranial level of his umbilicus in the plain phase (Fig. 1a). The tumor was located in the peripheral part of the mesentery of the jejunum and partly compressed the adjacent jejunum. In the enhanced phase, the tumor lacked contrast. The peripheral artery was shown to be involved, indicating that the tumor developed in the mesentery of the jejunum; however, the adjacent jejunum showed good enhancement (Fig. 1b). The oral side of the jejunum did not show dilatation. A lymphatic cyst diagnosis was highly suspected.

The patient's abdominal symptom passed without exacerbation, and a follow-up examination was conducted in March. One month later, he underwent a scheduled CT examination, and the mass did not appear to have increased in size (Fig. 2a). Enhancement revealed apparent invasion of the wall of the jejunum without ischemia of the adjacent jejunum or dilatation of the oral side of the bowel (Fig. 2b). Magnetic resonance imaging (MRI) showed intermediate intensity on T1-weighted imaging (T1WI) (Fig. 3a) and high intensity on T2-weighted imaging (T2WI) (Fig. 3c). Relatively low intensity was observed with enhancement using gadolinium diethylenetriaminepentaacetic acid (DTPA) contrast medium on T1WI (Fig. 3b). An accumulation of cystic lesions with a variety of sizes was clearly depicted on the coronal view on T2WI (Fig. 3e).

Because it was expected that depiction of the tumor would be made somewhat more difficult by its location and the body shape of the patient, we conducted a sonography examination in reference to the location and properties of the tumor determined by CT and MRI. The lesion showed a low echo; however, a clear image was not obtained.

CT performed by the previous physician and at our institution showed that the tumor was located in the peripheral part of the jejunal mesentery and exhibited slight enteric compression without dilatation of the oral jejunum but no rapid increase in size. The patient hesitated to agree to an immediate operation but did agree to a 1month observation period. Permeation of the jejunal wall was shown by a second CT examination at our institution performed 1 month later, and from these findings, we highly suspected that the lymphangioma was invading the jejunal wall and thus decided to perform surgery.

We made a preoperative diagnosis of lymphangioma invading the jejunal wall, and with the patient's informed consent, we performed an operation to relieve his symptoms and obtain a definitive diagnosis of the tumor in April.

The patient was placed in the supine position under general anesthesia. Small incisions were made along the superior and inferior border of the umbilicus and connected through the bottom of the umbilicus with an Sshape. The length of the wound was approximately 5 $\mathrm{cm}$. We placed the Lapprotector ${ }^{\mathrm{Tw}}$ (Model FF0707; 

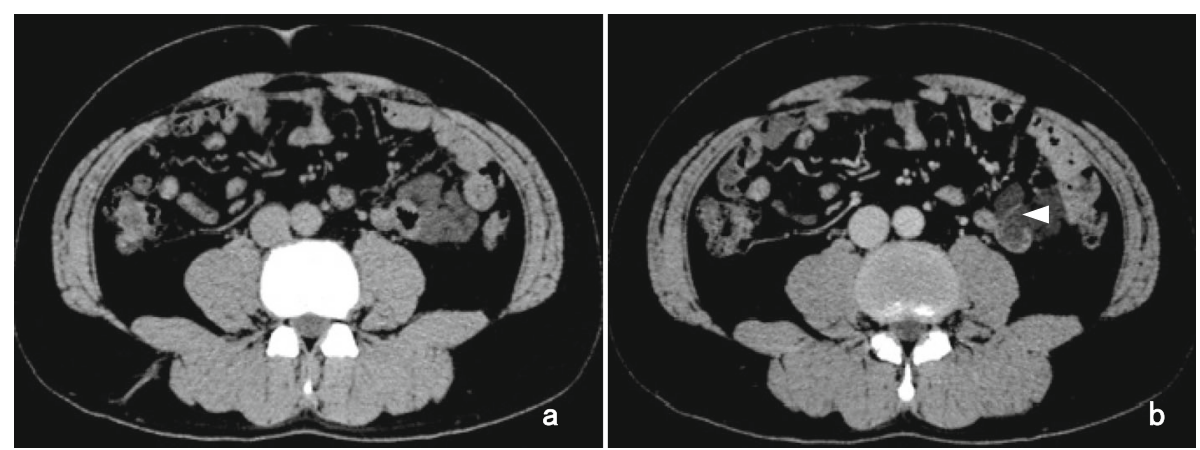

Fig. 1 Findings of the CT scan performed in February. a A low-density mass showing an irregular outline and measuring $45 \times 42 \mathrm{~mm}$ in size was detected; the mass was in contact with the jejunum in the left abdomen at the slightly cranial level of the umbilicus, as seen on plain CT. The oral side of the jejunum did not show dilatation. $\mathbf{b}$ In the contrast-enhanced image, the tumor lacked contrast. The tumor involved the peripheral artery (white arrowhead), indicating that the tumor developed in the mesentery of the jejunum; however, the adjacent jejunum showed good enhancement

Hakko Co., Ltd., Medical Device Division, Japan) in the umbilical wound and then attached the E.Z Access ${ }^{\mathrm{Tm}}$ device (E.Z Access for FF07; Hakko Co., Ltd., Medical Device Division, Japan). A 12-mm trocar was placed through the E.Z Access device, and pneumoperitoneum was established with carbon dioxide $\left(\mathrm{CO}_{2}\right)$ gas at 8 $\mathrm{mmHg}$ of pressure. An accessory trocar $(5 \mathrm{~mm})$ was placed on the right side of the $12-\mathrm{mm}$ trocar through the E.Z Access device. Using forceps, we removed the omentum from the small intestinal front and found a soft yellowish mass in the mesentery of the jejunum that had invaded the wall of the jejunum (Fig. 4a). We removed the tumor from the body through the umbilical wound, resected the surrounding mesentery and part of the jejunum, and performed anastomosis in a functional end-to-end manner (Fig. 4b). The total operative duration was $94 \mathrm{~min}$, and the intraoperative blood loss volume was $30 \mathrm{ml}$.

\section{Pathological findings}

Macroscopically, the external surface of the mass had a whitish-yellowish and lobulated appearance. The tumor protruded from both sides of the mesentery leaves and was surrounded by the mesenteric peritoneum. The tumor measured $50 \times 45 \mathrm{~mm}$ in size and was located in the peripheral part of the mesentery and adjacent to the jejunum (Fig. 5a). The small intestinal mucosal surface formed a hemispherical uplift approximately $24 \times 20 \mathrm{~mm}$ in size adjacent to the mesenteric lesion without contraction (Fig. 5b, c). The cut surface of the mass after fixation by formalin revealed multicystic lesions of various sizes containing a café-au-lait-colored milky fluid similar to chyle (Fig. 5d, e). Histologically, the cyst wall showed an attenuated endothelial lining, surrounding rich adipose tissue and scattered smooth muscle fibers (Fig. 6a, b). In addition, small lymphoid aggregates also appeared focally. Immunohistochemical staining showed that the flat endothelial cells associated with the cysts were positive for the endothelial markers CD31 (Fig. 6c) and factor VIII-related antigen (Fig. $6 \mathrm{~d}$ ), partially positive for the lymphatic endothelial marker D2-40 (Fig. 6e) and the undifferentiated pluripotent stem cell marker CD34 (Fig. 6f), and negative for cytokeratin (AE1/AE3) (Fig. 6g) and the mesothelial marker calretinin (Fig. 6h). Dilated lymphatic ducts were observed in the tumorous lesion of the mesentery and adjacent to this lesion in the

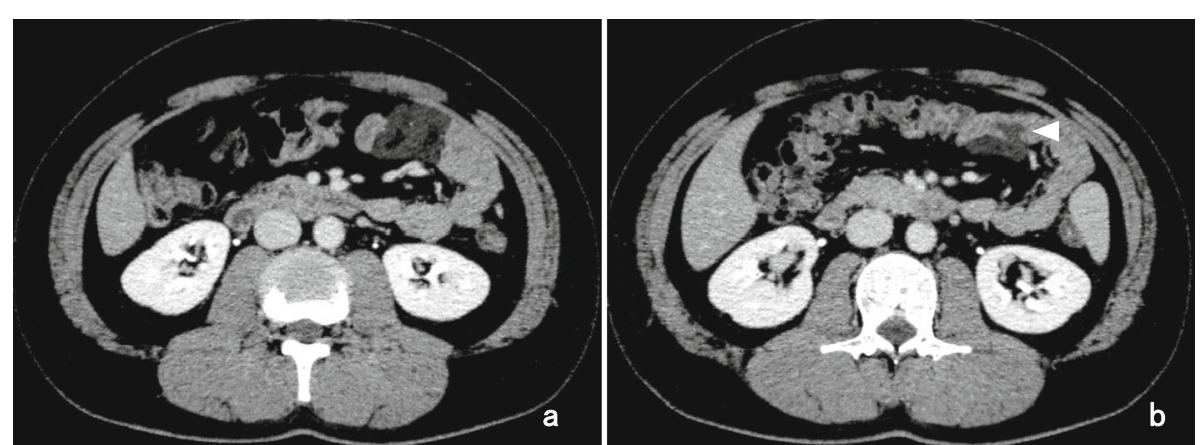

Fig. 2 Findings of the CT scan performed in March. a The low-density mass showed no increase in size, and the mass did not show contrast in the enhanced phase. Some lower-density components were observed inside of the low-density mass. $\mathbf{b} C T$ enhancement revealed apparent tumor invasion of the wall of the jejunum (white arrowhead) without ischemia in the adjacent jejunum or dilatation of the oral side of the bowel 


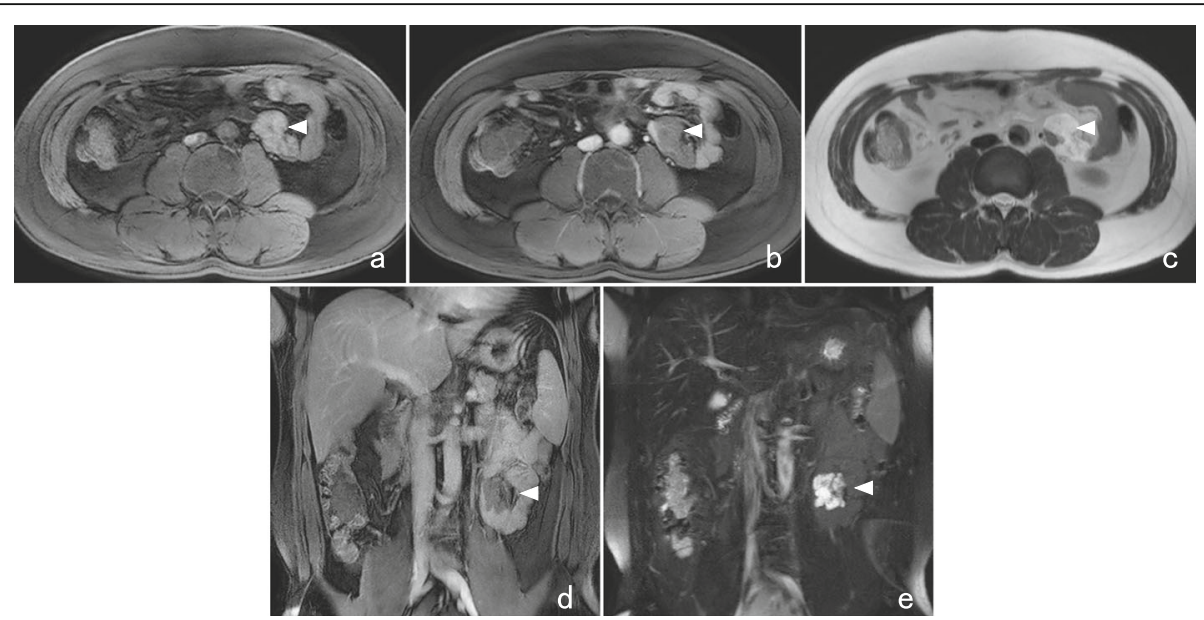

Fig. 3 MRI findings. a MRI showed the mass with intermediate intensity on T1WI (white arrowhead). $\mathbf{b}$ The enhanced phase on T1WI showed the mass with a relatively low intensity (white arrowhead). c On T2WI, the mass was depicted as a high-intensity tumor (white arrowhead). d Coronal view on T1WI. The irregularly shaped mass with a low signal intensity compressed the adjacent jejunum (white arrowhead). e Coronal view on T2WI. An accumulation of cystic lesions with a variety of sizes was more clearly depicted (white arrowhead)

muscularis propria and submucosal layer of the small intestine. In the wall of the jejunum without tumor invasion, dilation of the lymphatic ducts was not observed. These findings were consistent with cystic lymphangioma.

The patient recovered successfully and was discharged from the hospital 9 days after the operation.

\section{Discussion}

Lymphangiomas are congenital malformations of the lymphatic vessels and are usually associated with pediatric disease [9]. Sabin [10] divided the development of the lymphatic system into two stages: (1) the development of isolated lymph sacs derived from veins that are united by the thoracic duct and (2) the peripheral growth of lymph vessels that sprout from the endothelial lining of the sacs. The lymph sacs are transformed into the plexus or lymphatic capillaries by bridging the lumen with bands of connective tissue from which chains of lymph nodes are derived. Sabin [11] also reported that the lymphatic vessels were developed from the following five primordia: (1) paired jugular sacs lateral to the jugular veins, (2) an unpaired retroperitoneal sac at the mesenteric root, and (3) paired posterior sacs related to the sciatic veins (centrifugal theory). Godart [12] stated that the etiology of lymphangioma is related to abnormal development of the lymphatic system due to communication failure in a branch or branches within the central system, thus explaining why cystic lymphangiomas are found in the same position as fetal lymph sacs (centripetal theory). Approximately $95 \%$ of lymphangiomas are found in the head, neck, and axilla, and the other $5 \%$ occur in the mediastinum and abdominal cavity, including the mesentery, retroperitoneum, and bones [9]. It is presumed that there is a high association between developing lymphatic vessels and cystic lymphangioma. MLs account for less than 1\% of all lymphangiomas [3]. Abdominal lymphangiomas have been reported in the
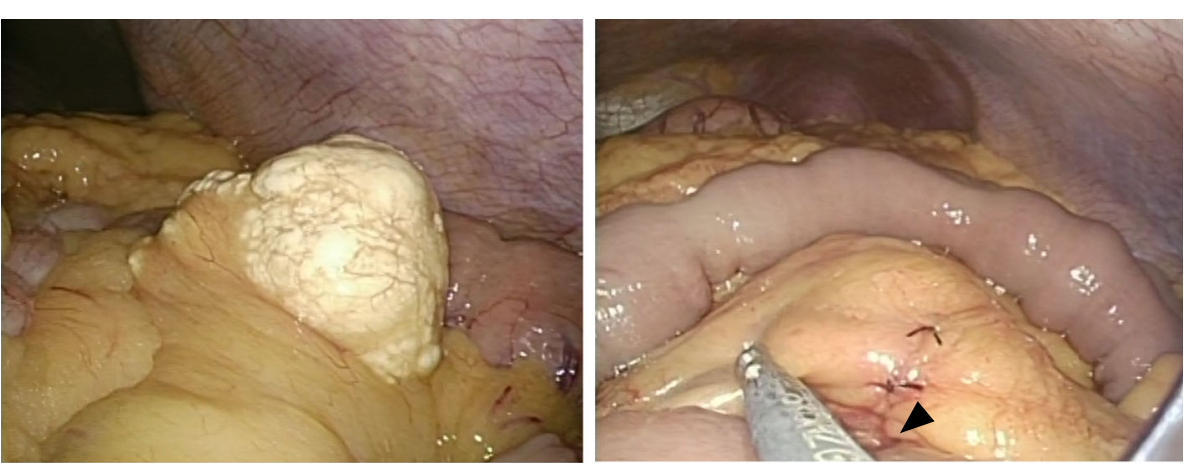

Fig. 4 Intraoperative photographs. a The omentum was removed from the front of the small intestine, and we found a soft yellowish mass in the mesentery of the jejunum that had invaded the wall of the jejunum. $\mathbf{b}$ We removed the tumor through the umbilical wound, resected the surrounding mesentery and part of the jejunum, and performed anastomosis in a functional end-to-end manner (arrowhead; anastomotic site) 


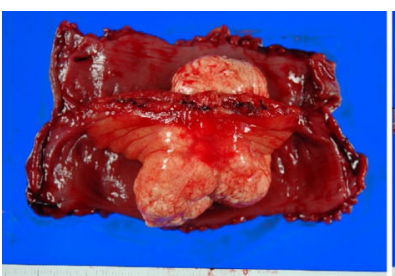

a

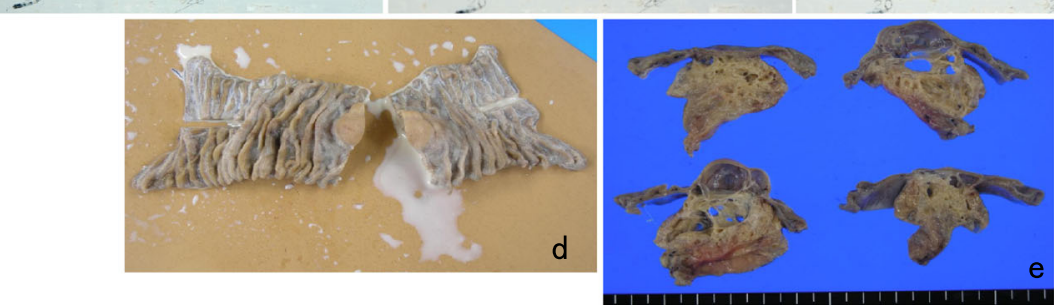

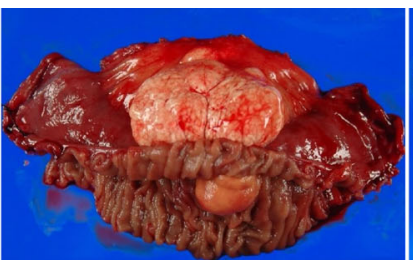

b

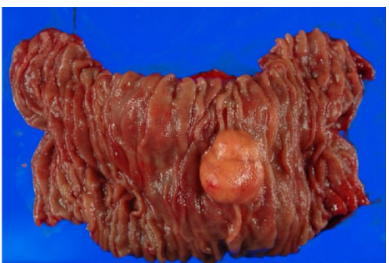

C

Fig. 5 Pathological findings. a Macroscopically, the external surface of the mass had a whitish-yellowish and lobulated appearance. The tumor protruded from both sides of the mesenteric leaves was surrounded by the peritoneum of the mesentery, measured $50 \times 45 \mathrm{~mm}$ in size, and was located in the peripheral part of the mesentery adjacent to the jejunum. b, c The small intestinal mucosal surface formed a hemispherical uplift approximately $24 \times 20 \mathrm{~mm}$ in size and was adjacent to the mesenteric lesion without contraction. $\mathbf{d}$ A formalin-fixed specimen. Café-au-laitcolored chyle-like milky fluid flowed out from inside the tumor after the tumor was cut. e The cut surface of the tumor showed multicystic lesions of various sizes without solid components or mural nodularity

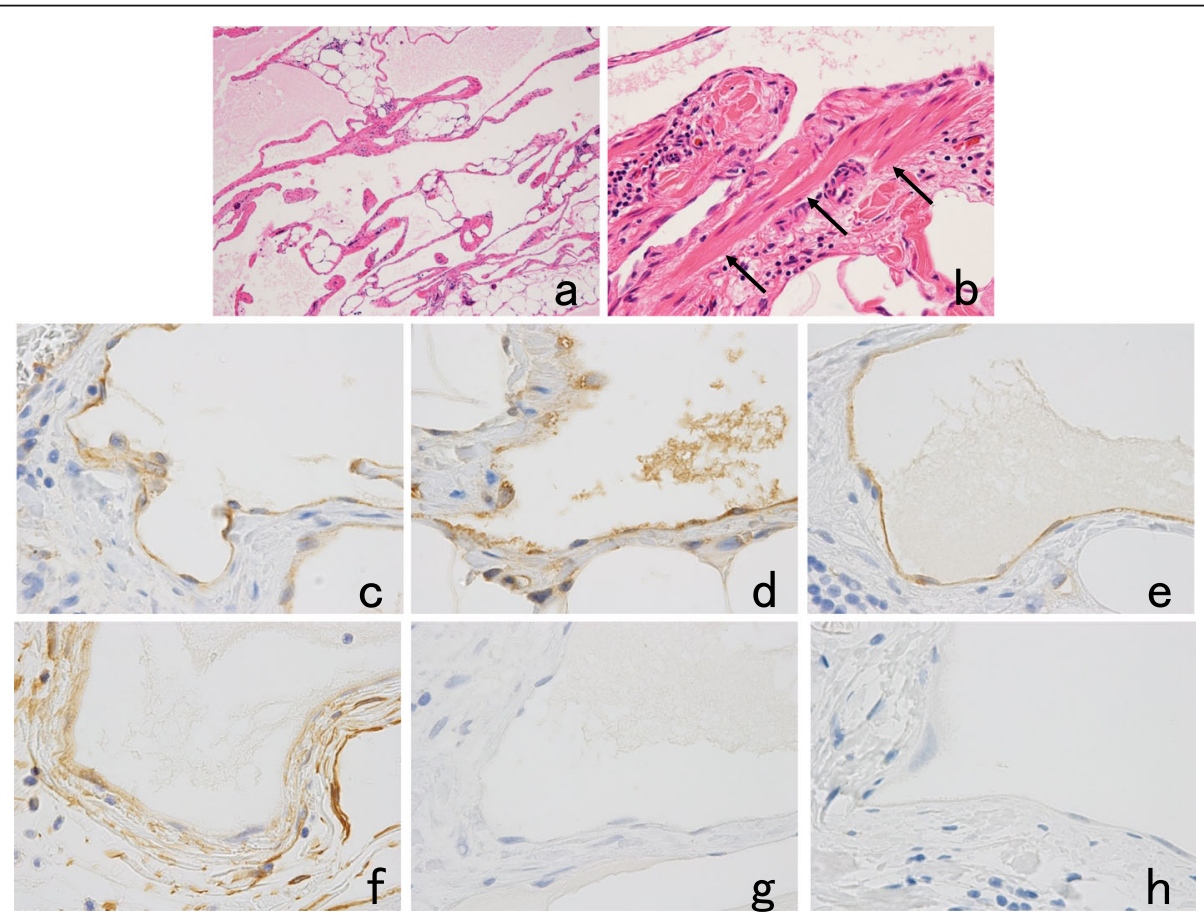

Fig. 6 Pathological findings. a, $\mathbf{b}$ Hematoxylin-eosin staining $(\mathbf{a} \times 40, \mathbf{b} \times 200)$. The cyst wall showed an attenuated endothelial lining, surrounding rich adipose tissue and scattered smooth muscle fibers (b arrows). Immunohistochemical staining showed that the flat endothelial cells were positive for the endothelial markers CD31 (c $\times 400)$ and factor VIII-related antigen $(\mathbf{d} \times 400)$, partially positive for the lymphatic endothelial marker D2-40 $(\mathbf{e} \times 400)$ and the undifferentiated pluripotent stem cell marker CD34 (f $\times 400)$, and negative for cytokeratin (AE1/AE3; $\mathbf{g}$ $\times 400)$ and the mesothelial marker calretinin $(\mathbf{h} \times 400)$ 
mesentery, accounting for more than half of the cases (58/ $107)$, followed by the retroperitoneal space $(11 / 107)$, spleen (9/107), colon (8/107), small intestine (8/107), pancreas (5/ 107), and other locations [13]. MLs result from a lack of communication between the small bowel lymphatic tissue and the main lymphatic vessels during fetal development, resulting in blind cystic lymphatic spaces lined by endothelial layers [14]. However, some lymphangiomas diagnosed in adults were assumed to be acquired and to have developed through different causes and pathways, such as obstruction of the existing lymphatic channels by an inflammatory fibrotic process, surgery, radiation exposure, abdominal trauma, or lymphatic hamartoma [1, 15-17]. Some reports have described no mesenteric masses in previous abdominal surgeries $[16,18]$. Although benign in nature, MLs may cause significant morbidity or mortality due to their large size and critical location. MLs can infiltrate the surrounding organs and cause potentially lifethreatening complications, such as traumatic rupture, anemia secondary to intraabdominal or intracavitary bleeding, ischemic tissue necrosis, intestinal gangrene secondary to volvulus, and intermittent intestinal obstruction [1]. One report described an ML lesion that encased the whole superior mesenteric artery [7]. MLs that occur in a relatively peripheral part of the mesentery, such as in our case, seemed to be diagnosed at a relatively early stage due to compression or invasion of the adjacent small bowel resulting in symptoms. The most common presentation is a freely mobile and nontender abdominal mass with partial small intestinal obstruction [7, 19], and tumor bleeding has rarely been reported [20]. However, this type of tumor could have a tendency to cause intestinal volvulus $[9,18,21,22]$.

Lymphangiomas are traditionally classified into three histological types: capillary (simple), cavernous, and cystic [8]. The capillary (simple) type usually originates in the skin and contains uniform, small, thin-walled lymphatic spaces. The cavernous type is composed of dilated lymphatic spaces of various sizes, is associated with lymphoid stroma, and generally maintains a connection with the adjacent normal lymphatic spaces. The cystic type consists of dilated lymphatic spaces of various sizes associated with collagen and smooth muscle bundles in the stroma but lacks connection to the adjacent normal lymphatic spaces [4, 23].

Differential diagnoses include a wide range of cystic intraabdominal lesions, such as mesenteric cysts, abdominal lymphomas, secondary metastases from an unknown primary tumor, tuberculosis, hydatid disease, small bowel adenocarcinomas, and rare mesenteric tumors, including desmoid tumors, schwannomas, smooth muscle tumors, sarcomas, cystic mesotheliomas, lymphangiosarcomas, and lymphangiomas with myxoid degeneration [17, 24].

To prove the lymphatic origin of the endothelial cells of cysts, we performed immunohistochemical analysis using a representative set of markers consisting of CD31, factor VIII-related antigen, D2-40, CD34, AE1/ $\mathrm{AE3}$, and calretinin for the diagnosis of lymphangioma [25]. Immunohistochemical staining of samples from the presented case showed that the flat endothelial cells associated with the cysts were positive for CD31 and factor VIII-related antigen, partially positive for D2-40 and $\mathrm{CD} 34$, and negative for cytokeratin (AE1/AE3) and calretinin. Factor VIII-related antigen, CD31 and CD34 can provide clues for the diagnosis of lymphangioma; however, both CD31 and CD34 label blood vessels and lymphatic endothelial tissue, making it difficult to differentiate lymphangioma from angioma [26]. D2-40 is a monoclonal antibody to the transmembrane mucoprotein, which is expressed by lymphatic endothelial cells, among others [26]. D2-40 shows immunoreactivity to only lymphatic endothelial tissue, so it is a marker specific for tissues of lymphatic origin [27]. Calretinin is a representative mesothelial marker, and reactivity to this marker can be used to distinguish disorders with a mesothelial cell origin, such as benign multicystic mesothelioma [23].

To treat ML, radical surgical excision is recommended, even when patients are asymptomatic, because ML can grow rapidly and invade adjacent structures, resulting in complications and the risk of sarcoma transformation upon irradiation [4]. However, radical excision can sometimes be technically impossible [4]. The recurrence rate has been reported to be $10 \%$ due to difficulty in resecting the entire cyst wall; the excision of retroperitoneal cysts is especially challenging due to their close proximity to vital retroperitoneal structures, making resection hazardous or even impossible [28, 29].

Minimally invasive surgery is a surgical treatment option. A relatively small ML, especially one that develops in the peripheral area of the mesentery, as in our case, is a good indication for laparoscopic-assisted surgery because the peripheral ML lesion can be easily removed from the body through a minimal wound and resection, and anastomosis can be performed under direct observation. A precise preoperative evaluation of the diagnostic images to determine the size, anatomical location, extent, and involved structures as well as the predicted laparoscopic surgical field aids in identifying an appropriate port site and wound length. Losanoff et al. [17] noted four types of MCLs: type 1, pedicled MCLs; type 2, sessile MCLs located in the mesenteric boundary; type 3, MCLs with retroperitoneal extension; and type 4, multicentric MCLs. MCLs located in the peripheral part of the mesentery were not included in this classification system. Kim et al. [6] presented a modified classification system for MLs consisting of four groups: group 1, MLs involving the intestinal wall; group 2, pedicled MLs with no relationship to the mesenteric vessels; group 3 , sessile MLs located in the mesenteric boundaries near the 
mesenteric vessels; and group 4, multicentric and diffusely infiltrated MLs. The lesion in our case was classified into group 1. Among the 25 cases in Kim's report, 14 were classified into group 1, and all of the lesions were excised with segmental resection of the intestine. The peripheral type of ML did not seem to be a rare type. However, there were no descriptions regarding the size of each tumor, and the number of cases limited to the peripheral part of the intestinal mesentery, apart from the root of the mesentery or main mesenteric vessels, was unknown. Of 14 patients, two patients in group 1 underwent laparoscopicassisted surgery. This classification system is useful for identifying the peripheral ML type as well as involvement of the intestinal wall, and considering the volume of the tumor, it could contribute to evaluating the indication for laparoscopic-assisted surgery.

We used a pressure of $8 \mathrm{mmHg}$ for pneumoperitoneum during the operation in this case. In our faculties, laparoscopic surgery begins with pneumoperitoneum at a pressure of $8 \mathrm{mmHg}$ to avoid referred pain by diaphragm hyperextension, symptoms of high carbon dioxide in the blood, atelectasis, and phlebothrombosis, and if a good visual field cannot be maintained, we increase the pneumoperitoneal pressure incrementally. Fortunately, we were able to accomplish the intended procedure with a sufficient surgical field of view at $8 \mathrm{mmHg}$ of pneumoperitoneal pressure in this case. However, if necessary, the pneumoperitoneal pressure can be increased to 12 or 15 $\mathrm{mmHg}$ to provide a better surgical field of view.

The Lapprotector ${ }^{\mathrm{Tm}}$ and E.Z Access ${ }^{\mathrm{mat}}$ devices are useful for laparoscopic-assisted tumor resection because they allow the use of multiple ports with minimal trauma and can also be used in single-port laparoscopic surgery (SPLS). We used two ports (one scope port and one forceps port) to remove the omentum. It seems that the excision of mesenteric tumors with a portion of the small intestine using a laparoscopic technique provides anatomical ease and the advantages of minimal surgical invasiveness, a reduced wound number and length, less postoperative pain, fewer wound-related complications, and improved cosmetic outcomes [30-32].

\section{Conclusions}

We report a case of MCL in an adult patient who was successfully treated by laparoscopic-assisted excision. ML should be considered in the differential diagnosis of patients with intraabdominal cystic masses. The optimal treatment is radical excision, even when the patient is asymptomatic. Laparoscopic-assisted tumor resection is a suitable surgical method for treating MLs located in the peripheral part of the mesentery.

\section{Abbreviations}

ALT: Alanine transaminase; BMl: Body mass index; CA125: Cancer antigen 125; CA19-9: Carbohydrate antigen 19-9; CD31: Cluster of differentiation 31;
CD34: Cluster of differentiation 34; CEA: Carcinoembryonic antigen; $\mathrm{CO}_{2}$ : Carbon dioxide; $\mathrm{CT}$ : Computed tomography; Gadolinium DTPA: Gadolinium diethylenetriaminepentaacetic acid; MCL: Mesenteric cystic lymphangioma; ML: Mesenteric lymphangioma; MRI: Magnetic resonance imaging; SPLS: Single-port laparoscopic surgery; T1WI: T1weighted imaging; T2WI: T2-weighted imaging; T-Bil: Total bilirubin; TChol: Total cholesterol; $\gamma$-GTP: $\gamma$-Glutamyl transferase

\section{Acknowledgements}

Not applicable.

\section{Authors' contributions}

HN drafted the manuscript, and TK performed the preoperative investigation. Al and TG provided academic advice. TT performed the operation. YS conducted the pathological examination. All authors have read and approved the final manuscript.

\section{Funding}

None of the authors have any funding to disclose.

\section{Availability of data and materials}

All data generated or analyzed during this study are included in this published article.

Ethics approval and consent to participate

All procedures used in this research were approved by the Ethics Committee of the National Hospital Organization.

\section{Consent for publication}

Written informed consent was obtained from the patient for the publication of this case report and any accompanying images. A copy of the written consent form is available for review by the Editor-in-Chief of this journal.

\section{Competing interests}

The authors declare that they have no competing interests.

\section{Author details}

${ }^{1}$ Department of Surgery, National Hospital Organization Tsuruga Medical Center, 33-1, Sakuragaoka, Tsuruga, Fukui 914-0195, Japan. ${ }^{2}$ First Department of Surgery, Faculty of Medicine, University of Fukui, 23-3,

Matsuokashimoaizuki, Eiheiji-cho, Yoshida-gun, Fukui 910-1193, Japan.

${ }^{3}$ Department of Human Pathology Kanazawa University Graduate School of Medicine, 13-1, Takara-machi, Kanazawa, Ishikawa 920-8640, Japan.

Received: 27 June 2019 Accepted: 20 September 2019

Published online: 24 October 2019

\section{References}

1. Chen J, Du L, Wan D-R. Experience in the diagnosis and treatment of mesenteric lymphangioma in adults: a case report and review of literature. World J Gastrointest Oncol. 2018;10:522-7.

2. Geraci G, Sciume C, Pisello F, Volsi FL, Facella T, Tinaglia D, et al. Mesenteric cyst lymphangioma; a case report and literature review. Ann Ital Chir. 2006; 77:521-7 discussion 528.

3. Roisman I, Manny J, Fields S, Shiloni E. Intra-abdominal lymphangioma. Br J Surg. 1989;76:485-9.

4. Siddique K, Bhandari S, Basu S. Giant mesenteric lymphangioma: a rare cause of a life-threatening complication in an adult. BMJ Case Rep. 2010; 2010. https://doi.org/10.1136/bcr.04.2010.2896

5. Losanoff JE, Kjossev KT. Mesenteric cystic lymphangioma: unusual cause of intra-abdominal catastrophe in an adult. Int J Clin Pract. 2005;59:986-7.

6. Kim SH, Kim HY, Lee C, Min HS, Jung SE. Clinical features of mesenteric lymphatic malformation in children. J Pediatr Surg. 2016;51:582-7.

7. Kumar B, Bhatnagar A, Upadhyaya VD, Gangopadhyay AN. Small intestinal lymphangioma presenting as an acute abdomen with relevant review of literature. J Clin Diagn Res. 2017;11:PD01-2.

8. Wegner G. Ueber Lymphangiome Arch Klin Chir 1877;127:203-8.

9. Jang JH, Lee SL, Ku YM, An CH, Chang ED. Small bowel volvulus induced by mesenteric lymphangioma in an adult: a case report. Korean J Radiol. 2009; 10:319-22. 
10. Sabin FR. The method of growth of the lymphatic system. In: The Harvey lectures 1915-1916. Philadelphia and London: J. B. Lippincott Company; 1916. p. $124-45$

11. Sabin FR. The lymphatic system in human embryos, with a consideration of the morphology of the system as a whole. Am J Anat. 1909;9:43-91.

12. Godart S. Embryological significance of lymphangioma. Arch Dis Child. 1966:41:204-6.

13. Levy AD, Cantisani V, Miettinen M. Abdominal lymphangiomas: imaging features with pathologic correlation. AJR Am J Roentogenol. 2004;182:1485-91.

14. Méndez-Gallart R, Solar-Boga A, Gómez-Tellado M, Somoza-Argibay I. Giant mesenteric cystic lymphangioma in an infant presenting with acute bowel obstruction. Can J Surg. 2009;52:E42-3.

15. Tung KSK, McCormack LJ. Angiomatous lymphoid hamartoma. Report of five cases with a review of the literature. Cancer. 1967;20:525-36.

16. Rojas CL, Molina GA. Lymphangioma cavernous of the small bowel mesentery, an infrequent cause of acute abdomen in adult. J Surg Case Rep. 2018;2018:riy018.

17. Losanoff JE, Richman BW, El-Sherif A, Rider KD, Jones JW. Mesenteric cystic lymphangioma. J Am Coll Surg. 2003;196:598-603.

18. Campbell WJ, Irwin ST, Biggart JD. Benign lymphangioma of the jejunal mesentery: an unusual cause of small bowel obstruction. Gut. 1991;32:1568.

19. Kosir MA, Sonnino RE, Gauderer NW. Pediatric abdominal lymphangiomas: a plea for early recognition. J Pediatr Surg. 1991;26:1309-13.

20. Xu X, Liu W, Zheng C. A rare cause of repeated gastrointestinal bleeding Mesenteric cavernous lymphangioma. Gastroenterology. 2014;146:e11-3.

21. Elukoti HN, Alcasoas S, Vernekar J, Hegde P, Pereira S. Mesenteric lymphangioma presenting as ileal volvulus. J Clin Diagn Res. 2015;9:TJ05-6.

22. Coulibaly Y, Keita S, Doumbia A, Togo A. Volvulus of the jejunum on cystic lymphangioma: about a clinical case. Afr J Paediatr Surg. 2016;13:95-7.

23. Suthiwartnarueput W, Kiatipunsodsai S, Kwankua A, Chaumrattanakul U. Lymphangioma of the small bowel mesentery: a case report and review of literature. World J Gastroenterol. 2012;18:6328-32.

24. Hureibi K, Sunidar OA. Mesenteric cystic lymphangioma mimicking malignancy. BMJ Case Rep. 2014. https://doi.org/10.1136/bcr-2014-203560

25. Gao L, Zhang S, Wang H, Qiu Y, Yang L, Yuan J, et al. Clinical and pathological characteristics of adrenal lymphangioma treated by laparoscopy via a retroperitoneal approach: experience and analysis of 7 cases. Int J Clin Med. 2015;8:4212-9.

26. Kim KH, Lee Jl, Bae JM. Significant growth of adrenal lymphangioma: a case report and review of the literature. Int J Surg Case Rep. 2015;17:48-50.

27. Ellis CL, Banerjee P, Carney E, Sharma R, Netto GJ. Adrenal lymphangioma: clinicopathologic and immunohistochemical characteristics of a rare lesion. Hum Pathol. 2011:42:1013-8.

28. Minocha PK, Roop L, Persad R. Cases of atypical lymphangiomas in children. Case Rep Pediatr. 2014;2014:626198. https://doi.org/10.1155/2014/626198.

29. Mendez-Gallart R, Bautista A, Estevez E, Rodriquez-Barca P. Abdominal cystic lymphangiomas in pediatrics: surgical approach and outcomes. Acta Chir Belg. 2011;111:374-7.

30. Hsu SJ, Chang YT, Chang MC, Yu SC, Lee JC, Shun CT, et al. Bleeding jejunal lymphangioma diagnosed by double-balloon enteroscopy. Endoscopy. 2007;39:E5-6.

31. Kenney B, Smith B, Bensoussan AL. Laparoscopic excision of a cystic lymphangioma. J Laparoendosc Surg. 1996;6:S99-101.

32. Kameda C, Miwa H, Kawabata R, Marukawa D, Murakami M, Noura S, et al. Laparoscopic resection of a jejunal inflammatory fibroid polyp that caused occult gastrointestinal bleeding, diagnosed via capsule endoscopy and double-balloon enteroscopy: a case report. Clin Endosc. 2018:51:384-7.

\section{Publisher's Note}

Springer Nature remains neutral with regard to jurisdictional claims in published maps and institutional affiliations.

Ready to submit your research? Choose BMC and benefit from:

- fast, convenient online submission

- thorough peer review by experienced researchers in your field

- rapid publication on acceptance

- support for research data, including large and complex data types

- gold Open Access which fosters wider collaboration and increased citations

- maximum visibility for your research: over $100 \mathrm{M}$ website views per year

At $\mathrm{BMC}$, research is always in progress.

Learn more biomedcentral.com/submissions 